\title{
Images - Adenomatoid tumor of testis, concerning for malignancy after ultrasound
}

\author{
Emily Nham ${ }^{1}$; Hamidreza Abdi $^{2}$; Eric Charles Belanger ${ }^{3}$; John Mahoney ${ }^{2}$ \\ ${ }^{1}$ Faculty of Medicine, University of Ottawa, Ottawa, ON, Canada; ${ }^{2}$ Division of Urology, Department of Surgery, \\ University of Ottawa, Ottawa, ON; Canada; ${ }^{3}$ Department of Pathology and Laboratory Medicine, University of \\ Ottawa, Ottawa, ON, Canada
}

Cite as: Can Urol Assoc J 2020 March 30; Epub ahead of print. http://dx.doi.org/10.5489/cuaj.6067

Published online March 30, 2020

$* * *$

\section{Introduction}

Adenomatoid tumors are rare benign tumors found in the genital tracts of males and females. These tumors arise from mesothothelial cell types and although rare, are the most common type of paratesticular tumor accounting for $30 \%$ of all paratesticular masses. ${ }^{1,2}$ These tumors are commonly found in the epididymis, although they may also be found in spermatic cord, tunica albuginea, and in rare cases the testicular parenchyma, prostate, ejaculatory ducts, and suprarenal glands..$^{3-6}$ Adenomatoid tumors are slow-growing and their growth is typically not associated with pain, however, there may be instances where testicular pain is present. ${ }^{8,9}$ Diagnosis of adenomatoid tumors may be particularly challenging to differentiate from other malignant testicular tumors based on clinical presentation and imaging alone.

\section{Case presentation}

A 39-year-old patient presented with a palpable mass on the left testicle and left testicular pain over one year, recently worse for the preceding 3 months. Physical examination revealed a $2 \mathrm{~cm}$ hard, tender, non-mobile and non-separable mass in the upper pole of the left testicle.

Examination of the scrotal sac was normal with no erythema or swelling. Serum levels of $\alpha$ fetoprotein (AFP) and $\beta$-human chorionic gonadotrophin ( $\beta-\mathrm{HCG}$ ) were 2.8 and $<5$, respectively and were within the normal ranges.

Ultrasound demonstrated a $13 \mathrm{~mm}$ well-demarcated hypoechoic solid mass in the left upper pole of the testicle, concerning for malignancy. A staging CT of the chest, abdomen and pelvis revealed no metastatic deposits. Given the suspicion of malignancy, a left radical orchiectomy was performed. The operative findings were consistent with a firm mass palpable at the upper pole of the left testicle, and successful removal of the left testicle and spermatic cord was performed through inguinal incision, with no complications. 
The final surgical pathology report revealed a well-circumscribed tumor predominantly within the epididymis abutting the testis parenchyma at the hilum. The tumor was composed of gland-like spaces lined by flat to cuboidal cells, and had a mixture of viable, inflamed and ischemic components (Figures 3 and 4). Infarction was evident at the centre of the lesion and was thought to explain the associated pain. Immunohistochemistry was performed which showed positive staining in the glandular structures for mesothelial-associated markers. The overall constellation of findings was found to be consistent with an adenomatoid tumor that underwent partial infarction. After one year of follow up, our patient is well with absence of recurrence.

\section{Discussion}

Adenomatoid tumors are rare paratesticular tumors that make up 2-3\% of all scrotal lesions. ${ }^{10}$ They typically present as small, firm, asymptomatic scrotal masses in the epididymis, however in exceptional cases may be identified in the testicular parenchyma and other scrotal structures. ${ }^{3-6}$ In these instances, a high degree of suspicion for malignant germ cell tumors must be maintained. Ultrasonography is considered first line for the investigation of testicular tumors as it can help identify the location of lesions, as well as other cystic, solid and vascular features. ${ }^{11}$ Specific features identified on ultrasound may facilitate testicular sparing surgery in benign tumors, however this is not always possible as demonstrated by this present case.

Our case presented with painful testicular mass and ultrasound study identified the tumor as being in the testicular parenchyma while pathology revealed a mass predominantly in the epididymis. The variable location and sonographic characteristics of adenomatoid tumors pose significant challenges in making benign diagnoses. As such, standard radical orchiectomy was performed due to clinical suspicion for malignancy. Given the lack of evidence for relapse or malignant transformation, serial follow up with tumor markers and imaging has not been recommended in the literature. 


\section{References}

1. Eble JN. Classification of tumours: pathology and genetics of tumours of the urinary system and male genital organs. World Health Organization classification of tumours 2004;255-7.

2. Delahunt B, Eble JN, King D, et al. Immunohistochemical evidence for mesothelial origin of paratesticular adenomatoid tumour. Histopathology 2000;36:109-15.

3. Di Pierro GB, Sciarra A, Innocenzi M, et al. Rare case of multiple adenomatoid tumors arising from tunica vaginalis of testis and epididymis. Actas Urol Esp 2010;34:560-1.

4. Monappa V, Rao ACK, Krishnanand G, et al. Adenomatoid tumor of tunica albuginea mimicking seminoma on fine needle aspiration cytology. Acta Cytol 2009;53:349-52.

5. Schwartz EJ, Longacre TA. Adenomatoid tumors of the female and male genital tracts express WT1. Int J Gynecol Pathol Off J Int Soc Gynecol Pathol 2004;23:123-8.

6. Alexiev BA, Xu LF, Heath JE, et al. Adenomatoid tumor of the testis with intratesticular growth: a case report and review of the literature. Int J Surg Pathol 2011;19:838-42.

7. Kassis A. Testicular adenomatoid tumours: clinical and ultrasonographic characteristics. BJU Int 2000;85:302-4.

8. Piccin A, Conneally E, Lynch M, et al. Adenomatoid tumor of the testis in a patient on imatinib therapy for chronic myeloid leukemia. Leuk Lymphoma 2006;47:1394-6.

9. Pacheco AJ, Torres JLM, de la Guardia FVD, et al. Intraparenchymatous adenomatoid tumor dependent on the rete testis: A case report and review of literature. Indian J Urol IJU J Urol Soc India 2009;25:126-8.

10. Urkmez A, Akan S, Ozsoy E, Sahin A, Koca O, et al. Diagnosis And Treatment Of Paratesticular Adenomatoid Tumors. J Coll Physicians Surg Pak 2018;28:S217-9. 


\section{Figures and Tables}

Fig. 1. Gray-scale ultrasound (A) sagittal and (B) transverse scans showing a $13 \mathrm{~mm}$ welldemarcated hypoechoic mass in the left upper pole of the testicle.

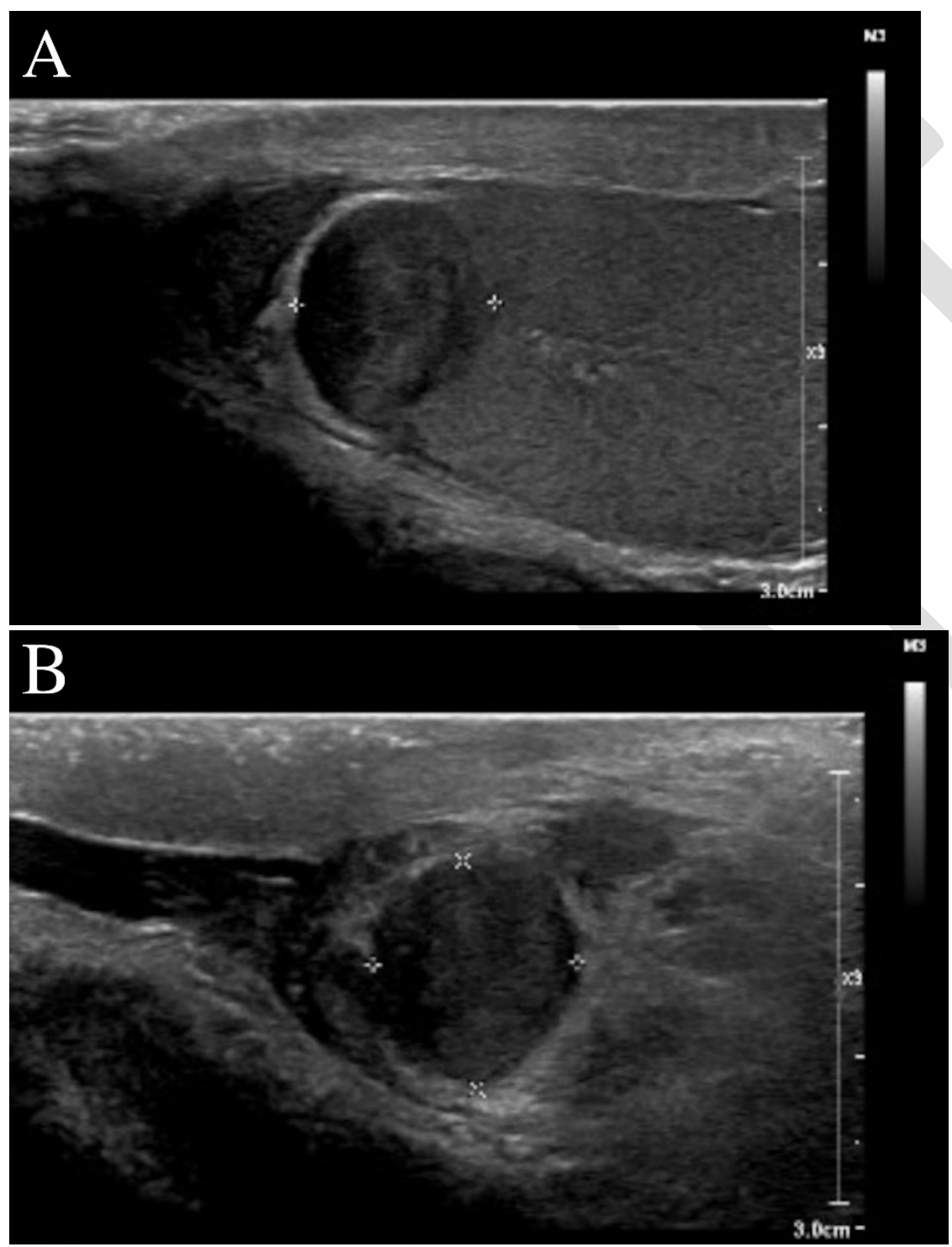


Fig. 2. Colour doppler ultrasound scan of left testicle showing a solid mass in the left upper pole of the testicle with limited vascularity.

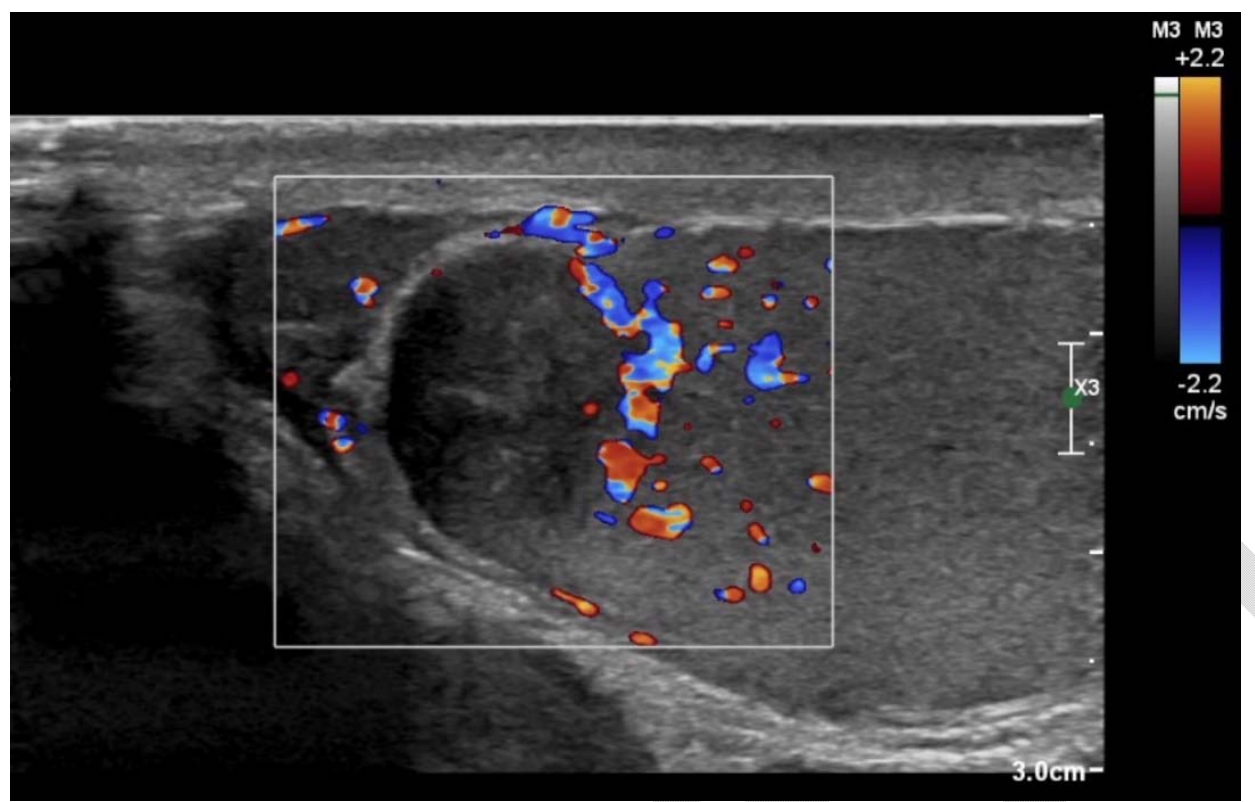

Fig. 3. Intermediate-power view of the adenomatoid tumor that displays a mixture of viable (right side), inflamed (middle) and ischemic (left side) components using hematoxylin and eosin stain (5.0X).

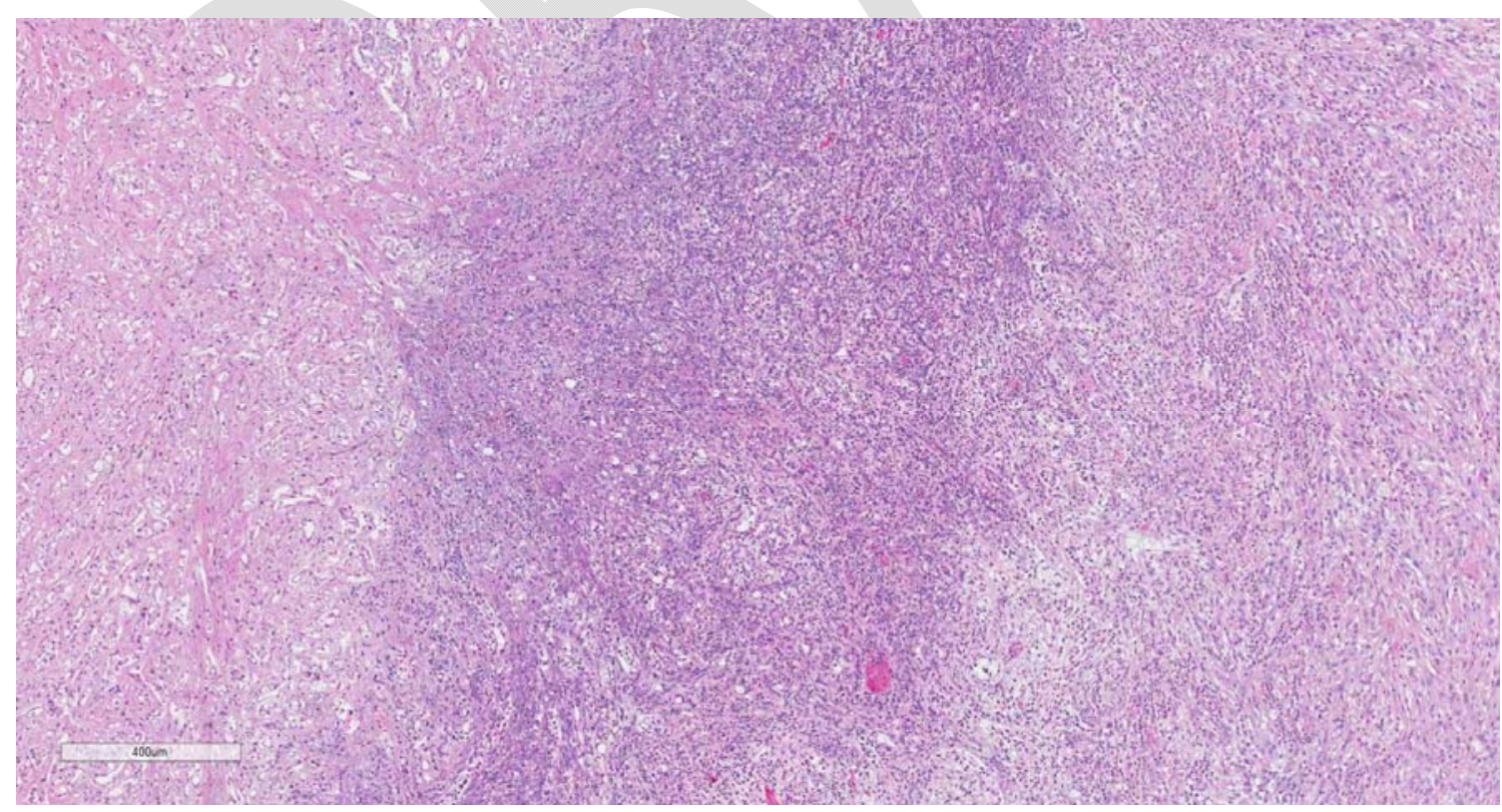


Fig. 4. Intermediate-power view of the adenomatoid tumor which displays tubular and glandular architectures and a mixture of viable (right side), inflamed (middle) and ischemic (left side) components using calretinin stain (5.0X). Given the ischemic nature of the tumor, the viable component is best highlighted on the calretinin stain which also best demonstrates the tubular and glandular architectures that are typically seen in these tumors.

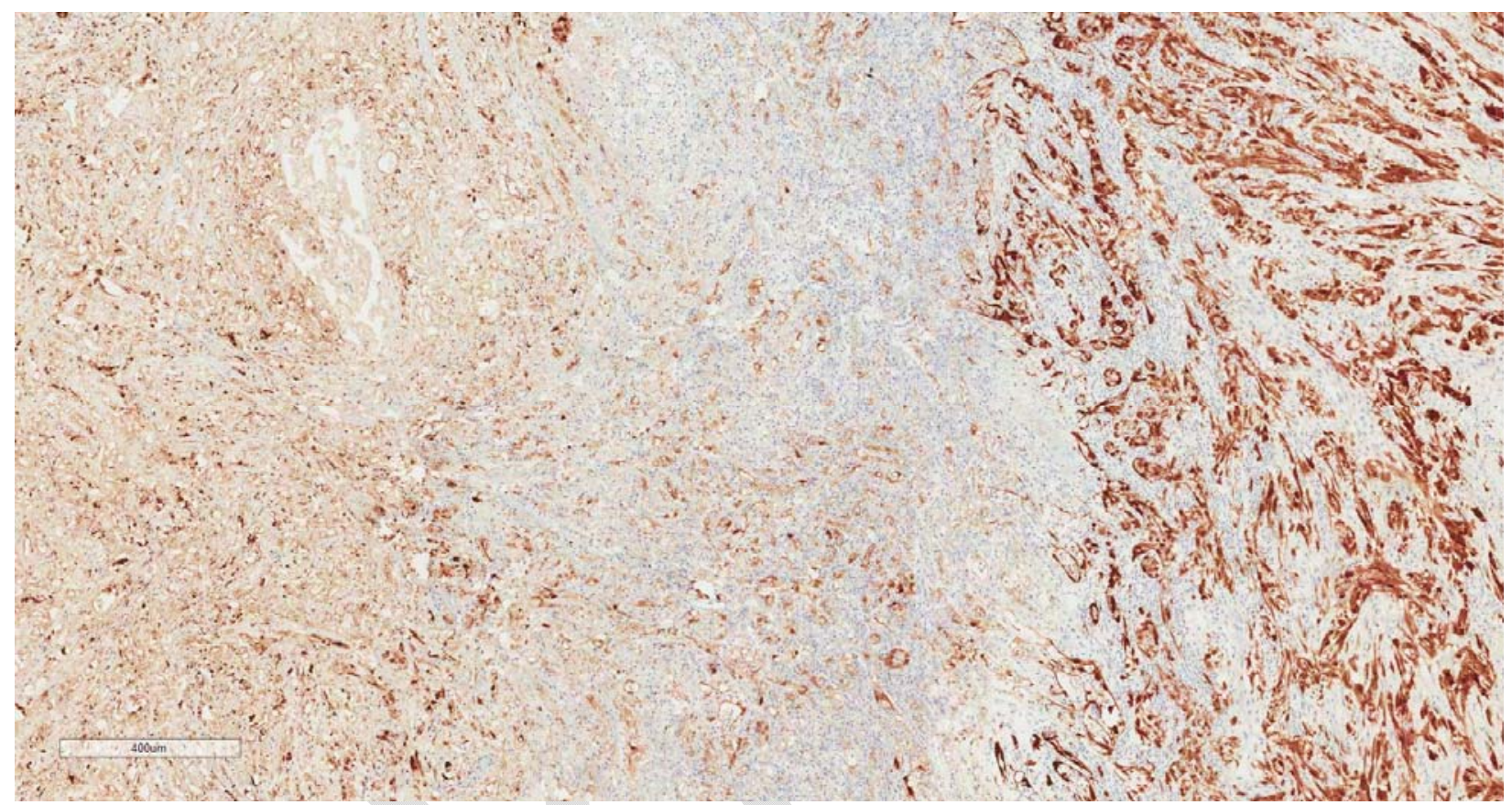

